\title{
TWO-TO-ONE TRANSFORMATIONS ON 2-MANIFOLDS
}

\author{
BY \\ VENABLE MARTIN AND J. H. ROBERTS
}

Introduction. An exactly 2-to-1 transformation is one for which every inverse image set consists of exactly 2 points. This notion was introduced by O. G. Harrold $\left({ }^{1}\right)$, who showed that no such continuous transformation could be defined over an arc. This result has been extended $\left({ }^{2}\right)$ to the case of the closed 2-cell. Further results concerning these transformations have been obtained by Harrold and by $\mathrm{P}$. W. Gilbert $\left({ }^{3}\right)$. The present paper is concerned with continuous 2-to-1 transformations defined on a compact 2-manifold, with or without bounding curves. The problem of the existence of such a transformation is solved, and the collection of all image spaces is determined. A precise statement of the main results is given below.

Throughout this paper the letter $M$ will be used to denote a compact 2-manifold (absolute), or else a compact 2-manifold with boundary, the boundary consisting of a finite number of mutually exclusive simple closed curves. The set $M$ will be considered as the whole space. $T$ will denote some exactly 2-to-1 continuous transformation defined over $M$. The set of inverse images under $T$ is $\left({ }^{4}\right)$ an upper semi-continuous collection $G$ filling $M$, and every element of $G$ is a pair of points. For each $x \varepsilon M$ let $s(x)$ be the point such that the pair $x, s(x)$ is an element of the collection $G$. Let $f(x)=\rho(x, s(x))$, where $\rho$ is the metric on $M$. Let $K$ denote the set of all points $x \varepsilon M$ at which $f$ is continuous, and let $L$ denote the subset of $K$ consisting of those points $x$ such that $f$ is continuous both at $x$ and at $s(x)$. If $x$ is a point, then $x^{\prime}$ will denote $s(x)$; and if $C$ is any point set, then $C^{\prime}$ will denote $s(C)$. A point set $C$ will be called integral if $C^{\prime}=C$.

The term $n$-cell $(n=0,1,2)$ will denote a closed $n$-cell except where the context indicates the contrary. If $\beta$ denotes a closed $n$-cell, then $\beta^{0}$ will denote the open $n$-cell whose closure is $\beta$. If $A$ denotes a complex, then $A^{*}$ denotes

Presented to the Society, February 24, 1940; received by the editors March 25, 1940.

(1) The non-existence of a certain type of continuous transformation, Duke Mathematical Journal, vol. 5 (1939), pp. 789-793. See also abstracts by Harrold in the Bulletin of the American Mathematical Society, vol. 46 (1940), pp. 43, 44.

(2) J. H. Roberts, Two-to-one transformations, Duke Mathematical Journal, vol. 6 (1940), pp. 256-262. This paper will be referred to hereafter as Transformations.

(3) See abstracts in the Bulletin of the American Mathematical Society, vol. 45 (1939), p. 835 , and vol. 46 (1940), pp. $42,43$.

(4) This follows readily from the compactness of $M$. However, the corresponding statement in Transformations does not follow from the continuity of $T$, and must be taken as an extra hypothesis in those theorems of that paper where $M$ was not assumed to be compact. This does not affect the main result of that paper. 
the point set covered by the complex $A$. If $A$ is a complex, then $\chi(A)$ will denote $-\alpha_{0}+\alpha_{1}-\alpha_{2}$, where $\alpha_{i}$ is the number of $i$-cells in $A$. This is the negative of the Euler characteristic as given by Alexandroff-Hopf $\left(^{5}\right)$. We assume a metric $\rho$ on $M$ having the following property: If $a$ and $b$ are points of $M$, then for any $\epsilon>0$ there is an arc $a b$ whose diameter is less than $\rho(a, b)+\epsilon$. If $\sigma$ is any metric on $M$, then a metric $\rho$ with the above property can be obtained by taking $\rho(a, b)$ equal to the g.l.b. of the diameters of arcs joining $a$ and $b$ in $M$.

The principal results of the paper are as follows:

$A$ necessary and sufficient condition that there be a $T$ defined over $M$ is that $\chi(M)$ be even. If $T(M)=B$, then $\chi(M)=2 \chi(B)$. Let $B_{k}$ denote a space which can be obtained from a compact manifold with $k$ bounding curves $(k=0,1,2, \cdots)$, by the identification by pairs of a finite number of interior points of the manifold. A compact manifold $M$ with $n$ bounding curves $(n=0,1,2, \cdots)$, and a space $B_{k}$ are said to be properly related if (1) $\chi(M)$ $=2 \chi\left(B_{k}\right)$ and (2) $\frac{1}{2} n \leqq k \leqq n$. Given a manifold $M$ and a space $B_{k}$, a necessary and sufficient condition that there be a $T$ defined over $M$ such that $T(M)=B_{k}$ is that $M$ and $B_{k}$ be properly related.

These results are obtained in Part II. In order to obtain these results, it is essential to determine the nature of the discontinuities of $f$ and the topological character of the set $M-K$. This is done in Part I.

\section{PART I}

Leмma 1. If the point $p$ is not on the boundary of $M$ (i.e., if $p$ has a 2-cell neighborhood), then $p$ is in $L$ if it is in $K$.

Proof. If $p$ is in $K$, then a sufficiently small open 2-cell containing $p$ is mapped topologically by $s$ into an open 2 -cell containing $s(p)$. Since an open 2 -cell in $M$ is necessarily open in $M$, and since $s$ has period 2, it follows that $s(p)$ is also in $K$, whence $p$ is in $L$.

TheOREM 1. The set $L$ is dense and open in $M$.

Proof. Since $K$ is dense and open (see Transformations), it follows from Lemma 1 that $L$ is dense. If $p$ is in $L$, then $p$ and $s(p)$ are in the open set $K$. In view of the upper semi-continuity of the collection $G$ it follows that if $x$ is sufficiently close to $p$ or to $s(p)$, then $x$ and $s(x)$ are in $K$, and therefore in $L$. Thus $L$ is open.

Lemma 2. If a simple closed curve $J$ bounds an open 2-cell $U$, and $R$ is any region which contains all of $J$ except possibly one point, then $R \cdot U$ is connected.

Proof. Let $x$ and $y$ be any two points of $R \cdot U$ and let $x y$ be an arc joining $x$ and $y$ in $R$. If $x y \cdot J=0$, then the arc $x y$ lies in $R \cdot U$. If $x y \cdot J \neq 0$, then let $z$

(5) Topologie I, Berlin, 1935, p. 214. 
and $w$ be points on $J$ and on the arc $x y$ in the order $x z w y$ (possibly $z=w$ ) such that no point of $J$ precedes $z$ or follows $w$ on the arc $x z w y$. In view of the hypothesis on $J$ there is an arc zvw belonging to $J \cdot R$. Since finally $R$ is open and contains zvw, there are points $z_{1}$ and $w_{1}$ on the arc $x y$ in the order $x z_{1} z w w w_{1} y$ and an arc $z_{1} w_{1}$ lying in $R \cdot U$. Then the arc $x z_{1}+z_{1} w_{1}+w_{1} y$ joins $x$ and $y$ and lies in $R \cdot U$. Since each pair $x, y$ of $R \cdot U$ lies on an arc in $R \cdot U$, this set is connected.

LemMA 3. If $H$ is a closed 2-cell in $M$, then $H$ does not contain $5 \operatorname{arcs}^{a} d_{i} a^{\prime}$ $(i=1, \cdots, 5)$ such that (1) each 2 of these arcs have only their end-points a and $a^{\prime}$ in common, (2) $\sum a d_{i} a^{\prime}$ is in $L$ except for the points $d_{3}, d_{4}$, and $d_{5}$, which are not in $K$, (3) $d_{2}=d_{1}^{\prime}$ and $s\left(a d_{1} a^{\prime}\right)=a^{\prime} d_{2} a$, and (4) for $i=3,4$, and 5, $s\left(a d_{i}-d_{i}\right)=a^{\prime} d_{i}-d_{i}$.

Proof. Suppose the lemma is false and there exist five $\operatorname{arcs} a d_{i} a^{\prime}$ in a closed 2-cell $H$ and properties (1), (2), (3) and (4) of the lemma hold. Now for $i=3,4$, or 5 , as $x \rightarrow d_{i}$ on the arc $a d_{i}$ the point $s(x) \rightarrow d_{i}$ on the arc $a^{\prime} d_{i}$. Let $J$ denote the simple closed curve $a d_{1} a^{\prime} d_{2} a$, and let $U$ denote its interior with respect to $H$. Let $R$ be the component of $L$ containing $J$. We consider three cases.

Case 1. At least two of the points $d_{3}, d_{4}, d_{5}$ are in $U$. Suppose that $d_{3}$ and $d_{4}$ are in $U$. Then the arc $a d_{3} a^{\prime}$ lies in $U+a+a^{\prime}$, and $U=U_{1}+U_{2}+a d_{3} a^{\prime}-a-a^{\prime}$, where $U_{1}$ and $U_{2}$ are open 2-cells bounded by $a d_{1} a^{\prime} d_{3} a$ and $a d_{2} a^{\prime} d_{3} a$, respectively. We suppose, without loss of generality, that $d_{4}$ is in $U_{2}$. Now let $R_{0}, R_{1}$, and $R_{2}$ denote respectively $R \cdot U, R \cdot U_{1}$, and $R \cdot U_{2}$. By Lemma 2 the sets $R_{0}, R_{1}$, and $R_{2}$ are connected. Furthermore, they are open subsets of the open 2-cell $U$. Hence $s\left(R_{0}\right), s\left(R_{1}\right)$, and $s\left(R_{2}\right)$ are connected open sets, since $s$ is topological over $R$, and an open 2-cell in $M$ is necessarily open in $M$. Since $s(J)=J$, and $s\left(a d_{i} a^{\prime}-d_{i}\right)=a^{\prime} d_{i} a-d_{i}(i=3,4,5)$, it follows that $s\left(R_{0}\right), s\left(R_{1}\right)$, and $s\left(R_{2}\right)$, respectively do not intersect the boundary of $U, U_{1}$ and $U_{2}$. That is, either $s\left(R_{0}\right) \subset U$ or else $s\left(R_{0}\right)$ is in $M-\bar{U}$. But the second possibility is ruled out since $s\left(a d_{4} a^{\prime}-d_{4}\right)=a d_{4} a^{\prime}-d_{4}$, and this set is in $R_{0}$. Therefore $s\left(R_{0}\right) \subset U$. Likewise $s\left(R_{2}\right) \subset U_{2}$, for $d_{4}$ is in $U_{2}$. But now let $x_{1}, x_{2}, \cdots$ be points in $R_{2}$ such that $x_{n} \rightarrow d_{2}$. Since $d_{2}$ is in $L$ and $s\left(d_{2}\right)=d_{1}, s\left(x_{n}\right) \rightarrow d_{1}$. But $s\left(x_{n}\right) \varepsilon U_{2}$ and $d_{1}$ is not in $\bar{U}_{2}$. This is a contradiction.

Case 2. Exactly one of the points $d_{3}, d_{4}, d_{5}$ is in $U$. Suppose that $d_{5} \varepsilon U$ and $d_{3}$ and $d_{4}$ are not in $U$. The sum of $a d_{3} a^{\prime}$ and one of the two arcs $a d_{1} a^{\prime}$, $a d_{2} a^{\prime}$ is a simple closed curve bounding a 2 -cell $U_{1}$ which lies in $H$ and contains the other of these arcs, except for end-points. We suppose that the boundary of $U_{1}$ is $a d_{3} a^{\prime} d_{2} a$, and let $U_{2}$ be the 2-cell in $H$ bounded by $a d_{3} a^{\prime} d_{1} a$. To summarize, $H \supset \bar{U}_{1}$, and $U_{1}=U+U_{2}+a d_{1} a^{\prime}-a-a^{\prime}$. Let $R_{0}, R_{1}$, and $R_{2}$ denote respectively $R \cdot U, R \cdot U_{1}$, and $R \cdot U_{2}$. Then it follows that $s\left(R_{0}\right) \subset U$ and $s\left(R_{1}\right) \subset U_{1}$, since both $U$ and $U_{1}$ contain the set $a d_{5} a^{\prime}-a-a^{\prime}$, and $s\left(a d_{5} a^{\prime}-d_{5}\right)=a d_{5} a^{\prime}-d_{5}$. Now let $x_{1}, x_{2}, \cdots$ be points in $R_{2}$ converging to $d_{1}$. 
Then $s\left(x_{1}\right), s\left(x_{2}\right), \cdots$ are points in $U_{1}$ (for $R_{2} \subset R_{1}$ ) converging to $d_{2}$. But for $n$ sufficiently large $s\left(x_{n}\right)$ is in $U$, hence in $R_{0}$. But $s\left(R_{0}\right) \subset U$, and $s\left(s\left(x_{n}\right)\right)=x_{n}$. Thus $x_{n}$ is in both $U$ and $U_{2}$ and we have a contradiction.

Case 3. None of the points $d_{3}, d_{4}, d_{5}$ is in $U$. Then there is a 2-cell in $H$ bounded by the sum of one arc from the set $\left\{a d_{i} a^{\prime}\right\}(i=1,2)$ and one arc from the set $\left\{a d_{i} a^{\prime}\right\}(i=3,4,5)$, and containing one of $d_{1}, d_{2}$, and one of $d_{3}, d_{4}, d_{5}$. For definiteness we may suppose there are 2-cells $U, U_{1}$, and $U_{2}$ bounded respectively by curves $a d_{1} a^{\prime} d_{2} a, a d_{3} a^{\prime} d_{2} a$, and $a d_{3} a^{\prime} d_{1} a$, such that $d_{4}$ is in $U_{2}$ and $U_{1}=U+U_{2}+a d_{1} a^{\prime}-a-a^{\prime}$. Let $R_{2}=R \cdot U_{2}$. Since $d_{4}$ is in $U_{2}$, it follows by earlier arguments that $s\left(R_{2}\right) \subset U_{2}$. Let $x_{1}, x_{2}, \cdots$ be points in $R_{2}$ converging to $d_{1}$. Then $s\left(x_{n}\right) \rightarrow d_{2}$. But $d_{2}$ is not in $\bar{U}_{2}$ while $s\left(x_{n}\right) \subset U_{2}$. This contradiction completes the proof of the lemma

Lemma 4. Suppose $q$ is a point, $H$ is a closed 2-cell, $V$ is an open set, and $\epsilon$ is a positive number, and the following properties hold:

(1) $H$ contains a neighborhood of $q$;

(2) $V \supset q$;

(3) $q$ is a limit point of $M-K$, and

(4) if $p \varepsilon K \cdot V, f(p)<\epsilon$, and $p t$ is any arc in $V$ and in $K+t$, where $t$ is not in $K$, then $f(x) \rightarrow 0$ as $x \rightarrow t$ on the arc $p t$.

Then it follows that there exists an $\epsilon_{1}>0$ such that no arc cc' lies in $K \cdot S\left(q, \epsilon_{1}\right)$.

Proof. Suppose the lemma is false. Then for each positive integer $n$ there is an $\operatorname{arc} c_{n} c_{n}^{\prime}$ which is a subset of each of the sets $H, K, V$, and $S(q, 1 / n)$, and for $n$ sufficiently large the arc $s\left(c_{n} c_{n}{ }^{\prime}\right)$ is in $H \cdot K \cdot V$. The set $c_{n} c_{n}^{\prime}+s\left(c_{n} c_{n}^{\prime}\right)$ contains $\left({ }^{6}\right)$ a simple closed curve $J$, the sum of two arcs $u t u^{\prime}$ and $u^{\prime} t^{\prime} u$ such that $s\left(u t u^{\prime}\right)=u^{\prime} t^{\prime} u$. Then $J$ bounds an open 2 -cell $H_{1}$ which is a subset of $H$. Let $R$ be the component of $K$ which contains $J$. Then by Lemma $2 R \cdot H_{1}$ is connected. We want to get a simple closed curve $a d_{1} a^{\prime} d_{2} a$ lying in $R \cdot H_{1}$ and such that $s\left(a d_{1} a^{\prime}\right)=a^{\prime} d_{2} a$. This will follow readily if we prove that $s\left(R \cdot H_{1}\right)=R \cdot H_{1}$, for then any point $p$ in this set can be joined to $p^{\prime}$ by an arc $p p^{\prime}$ in this set, and some subset of $p p^{\prime}+s\left(p p^{\prime}\right)$ will be the desired curve. If there is no point of $M-K$ in $H_{1}$, then $s$ is topological over $\bar{H}_{1}$, and $s\left(\bar{H}_{1}\right)$ is a closed 2-cell with $J$ for boundary. Either $s\left(H_{1}\right) \subset H_{1}$ or $s\left(H_{1}\right) \subset M-\bar{H}_{1}$. But the first possibility is ruled out, because under it $s$ is a topological mapping of the closed 2-cell $\bar{H}_{1}$ into itself which has no fixed point: Under the second possibility $\bar{H}_{1}+s\left(\bar{H}_{1}\right)$ is a sphere, hence is $M$. But $\bar{H}_{1}+s\left(\bar{H}_{1}\right)$ is in $K$, contrary to the fact that there are points in $M-K$ (e.g., the point $q$ ). Thus there is some point of $M-K$ in $H_{1}$. Join a point $p$ of $R \cdot H_{1}$ to a point $t$ of $M-K$ in $H_{1}$ by an arc $p t$ lying in $H_{1}$. Then $f(x) \rightarrow 0$ as $x \rightarrow t$ on the arc $p t$, and therefore for $x$ near enough to $t, s(x)$ is also in $H_{1}$. Hence $s\left(R \cdot H_{1}\right) \subset H_{1}$.

Now by Lemma $1, s\left(R \cdot H_{1}\right) \subset K$. It follows that $s\left(R \cdot H_{1}\right)=R \cdot H_{1}$, and the desired simple closed curve exists. That is, there is a simple closed curve

(6) See Transformations, $\S 8$, for a proof. 
$a d_{1} a^{\prime} d_{2} a$ lying in $H_{1}$ and in $K$, and such that $s\left(a d_{1} a^{\prime}\right)=a^{\prime} d_{2} a$. By Lemma 1 , this curve lies in $L$. There is an arc joining $a$ to some point of $M-K$, such that this arc is in $H \cdot V$, has only the point $a$ on the simple closed curve $a d_{1} a^{\prime} d_{2} a$, and has no point, except possibly an end-point, on the boundary of $M$. On this arc let $d_{3}$ be the first point of $M-L$. Then $d_{3}$ is in $M-K$. For if $d_{3}$ is the other end-point it is by definition in $M-K$. In the other case $d_{3}$ is not on a bounding curve of $M$ and hence by Lemma 1 is in $M-K$ if it is in $M-L$. Then $f(x) \rightarrow 0$ as $x \rightarrow d_{3}$ on the arc $a d_{3}$. It follows from Theorem 3 of Transformations $\left({ }^{7}\right)$ that $a d_{3}+s\left(a d_{3}-d_{3}\right)$ contains two arcs, atd $d_{3}$ and $a^{\prime} t^{\prime} d_{3}$ which have only $d_{3}$ in common and such that $s\left(a t d_{3}-d_{3}\right)=a^{\prime} t^{\prime} d_{3}-d_{3}$. Denote the sum $a t d_{3}+d_{3} t^{\prime} a^{\prime}$ by $a d_{3} a^{\prime}$.

In a similar way we obtain successively arcs $a d_{4} a^{\prime}$ and $a d_{5} a^{\prime}$ such that for $i=4,5, d_{i}$ is in $M-K$ but $a d_{i} a^{\prime}-d_{i}$ is in $L, a d_{i} a^{\prime}$ has only $a$ and $a^{\prime}$ in common with the sum of the other four arcs $a d_{j} a^{\prime}$, and $s\left(a d_{i}-d_{i}\right)=a^{\prime} d_{i}-d_{i}$. But then the five arcs $a d_{i} a^{\prime}(i=1, \cdots, 5)$ have the properties stated in Lemma 3, and we have reached a contradiction.

Lemma 5. Suppose $H$ is a closed 2-cell which contains a neighborhood $V$ of a point $q, p q$ is an arc in $V$ and in $L+q$ such that $f(x) \rightarrow 0$ as $x \rightarrow q$ on the arc $p q$. Let $R$ be the component of $L \cdot V$ which contains $p$, and suppose $R \cdot s(R) \cdot V=0$. Let $\epsilon$ be any positive number. Then there exists in $V$ an open set $W$ with boundary $J$ such that

(1) $W \supset q$ and $W+J$ is of diameter less than $\epsilon$, and $\bar{W}$ is a closed 2-cell;

(2) if $q$ is on a bounding curve of $M$, then $J$ is an arc $x a x^{\prime}$, where $x$ and $x^{\prime}$ are on a bounding curve of $M, a$ is in $M-K, x a-a$ is in $R$, and $s(x a-a)$ $=x^{\prime} a-a$;

(3) if $q$ is not on a bounding curve of $M$, then $J$ is a simple closed curve $a x b x^{\prime} a$, where $a$ and $b$ are in $M-K, a x b-a-b$ is in $R$, and $s(a x b-a-b)$ $=a x^{\prime} b-a-b$.

Proof. The proof given for Theorem 5 of Transformations requires only a trivial change in order to apply here.

Lemma 6. Suppose $q_{1}$ is a point, $H$ is a closed 2-cell, $V$ is an open set and $c q_{1}$ is an arc, and the following properties hold:

(1) $H \supset V \supset c q_{1}$;

(2) if $x \varepsilon H$, then either $f(x)<\epsilon$ or $f(x)>3 \epsilon$, where $4 \epsilon=f\left(q_{1}\right)$;

(3) $c \varepsilon$ L and $f(c)<\epsilon$;

(4) there is no arc connecting any point $d$ to $d^{\prime}$ and lying in $V \cdot K$; and

(5) if ef is any arc lying in $V \cdot L+f$, where $f(e)<\epsilon$ and $f$ is not in $K$, then $f(x) \rightarrow 0$ as $x \rightarrow f$ on the arc ef.

Then there exists an arc from $c$ to $q_{1}$ and lying in $V \cdot L+q_{1}$.

(7) Theorem 3 of Transformations is false as stated. The proof given is based on the assumption that $K$ is an integral set, i.e., that $s(K)=K$. Now $L$ is an integral set, and the argument given suffices to prove the theorem as stated if $K$ is replaced by $L$. In our application the the arc $a d_{3}$ lies in $L+d_{3}$, hence the modified theorem applies. 
Proof. Let $R_{1}$ be the component of $L \cdot V$ that contains $c$. Then $R_{1} \cdot s\left(R_{1}\right)=0$. Let $E$ denote the set of all $x$ in $R_{1}+s\left(R_{1}\right)$ for which $f(x)<\epsilon$. Let $t$ be the last point of $\bar{E}$ on the arc $c q_{1}$.

Suppose that $t$ is not accessible from $R_{1}$ by an arc $c t$ such that $f(x) \rightarrow 0$ as $x \rightarrow t$ on $c t$. Then $t$ is not accessible by any arc $u t$ lying in $R_{1}+s\left(R_{1}\right)+t$ and containing a point of $E$. Then we will show that there is an infinite sequence $c_{1}, c_{2}, c_{3}, \cdots$ such that (1) $c_{n} \varepsilon R_{1}$ and $f\left(c_{n}\right)<\epsilon$, (2) $c_{n} \rightarrow t$ as $n \rightarrow \infty$, and (3) there is a fixed positive $\delta$ such that every arc joining $c_{i}$ and $c_{j}(i \neq j)$ in $R_{1}$ has diameter greater than $\delta$. To prove this assertion consider the following hypothesis:

Given any positive number $\beta$ and any component $R_{\beta}$ of $R_{1} \cdot S(t, \beta)$ having $t$ on its boundary, it is true that for every $k$ there is some component of $R_{\beta} \cdot S(t, 1 / k)$ which has $t$ on its boundary. If this is true, then it follows that $t$ is accessible from $R_{1}$ by an arc $c t$ such that $f(x) \rightarrow 0$ as $x \rightarrow t$ along $c t$. But this contradicts a supposition made above. Hence the above hypothesis is false. This means that there is a positive number $\beta$ such that there is an infinite set $R_{1}^{1}, R_{1}^{2}, R_{1}^{3}, \ldots$ of components of $R_{1} \cdot S(t, \beta)$ such that, for every $i, t$ is not a limit point of $R_{1}^{i}$, but $t$ is a limit point of $\sum_{i=1}^{\infty} R_{1}^{i}$. And this implies that the sequence $c_{1}, c_{2}, c_{3}, \cdots$ exists.

There exist three open sets $W_{1}, W_{2}$, and $W_{3}$ containing $t$ and bounded respectively by $P_{1}, P_{2}$, and $P_{3}$, these being simple closed curves or $\operatorname{arcs}\left({ }^{8}\right)$, and there is an integer $N$, such that

(1) $V \supset \bar{W}_{1}$ and $W_{i} \supset \bar{W}_{i+1}(i=1,2)$;

(2) if $n>N$, there is an arc $c_{n} d_{n} e_{n}$ in $R_{1}$ and in $\bar{W}_{1}$, where $e_{n}$ and $d_{n}$ are on the boundaries of $W_{1}$ and $W_{2}$, respectively, and $c_{n}$ is in $W_{3}$; and

(3) if $n>N, m>N$, and $n \neq m$, then no component of $R_{1} \cdot \bar{W}_{1}$ contains both $c_{n}$ and $c_{m}$.

Suppose $n>N$. Let $x_{n}$ and $y_{n}$ be the first points of the boundary of $R_{1}$ on the circle $P_{2}$ starting from $d_{n}$ in the two senses, and let $x_{n} d_{n} y_{n}$ denote the indicated arc of the circle $P_{2}$. Then $s\left(x_{n} d_{n} y_{n}-x_{n}-y_{n}\right)+x_{n}+y_{n}$ is an arc $x_{n} d_{n}^{\prime} y_{n}$ in $s\left(R_{1}\right)+x_{n}+y_{n}$. Since $x_{n} d_{n}^{\prime} y_{n}$ does not intersect $c_{n} d_{n} e_{n}$ (because $\left.R_{1} \cdot s\left(R_{1}\right)=0\right)$, there is a positive $\gamma$ independent of $n$ such that $d\left(x_{n} d_{n}^{\prime} y_{n}\right)>\gamma$. But $d\left(x_{n} d_{n} y_{n}\right) \rightarrow 0$ as $n \rightarrow \infty$. If we drop to a subsequence, we may suppose $\lim \sup _{n \rightarrow \infty} x_{n} d_{n} y_{n}$ is a point $r$. Then $\lim \sup _{n \rightarrow \infty} x_{n} d_{n}^{\prime} y_{n}$ is contained in $r+s(r)$. But this is clearly impossible, and we have thus proved that $t$ is accessible by an arc $c t$ lying in $R_{1}+t$, and therefore in $V \cdot L+t$. It follows that $f(x) \rightarrow 0$ as $x \rightarrow t$ on the arc $c t$.

Suppose now that $t \neq q_{1}$. Choose $\epsilon_{1}<\rho\left(t, q_{1}\right)$. Let $W$ and $J$ be sets given by Lemma 5, where $t$ and the arc $c t$ replace $q$ and the arc $p q$, and $\epsilon_{1}$ replaces $\epsilon$. Then the arc $t q$ must contain a point $r(r \neq t)$ on $J$. But $J$ lies in $\bar{E}$, and $t$ is the last point of $\bar{E}$ on the arc $c q_{1}$. This contradiction proves Lemma 6 .

${ }^{8}$ ) If $t$ is on a bounding curve of $M$, then $P_{i}$ is an arc with end-points on this bounding curve. 
Theorem 2. If $q$ is a point in $M-K$ and $p q$ is an arc in $K+q$, then $f(x) \rightarrow 0$ as $x \rightarrow q$ on the arc $p q$.

Proof. We suppose the theorem is false. Then there is an arc $p q$ lying in $K+q, q$ not in $K$, such that $f(x) \rightarrow f(q)$ as $x \rightarrow q$ on the arc $p q$. Then by Theorem 4 of Transformations there is an open set $\dot{U}$, an arc $p_{1} q_{1}$, and a positive $\epsilon$, such that

(1) $U \supset q_{1}$;

(2) $p_{1} q_{1}-q_{1}$ is in $K$, but $q_{1}$ is not in $K$;

(3) $f(x) \rightarrow f\left(q_{1}\right)=4 \epsilon$ as $x \rightarrow q_{1}$ on the arc $p_{1} q_{1}$;

(4) if $x \varepsilon U$, then either $f(x)<\epsilon$ or $f(x)>3 \epsilon$;

(5) if $p_{2} q_{2}$ is any arc in $U \cdot\left(K+q_{2}\right)$, and $q_{2}$ is not in $K$, and if $f\left(p_{2}\right)<\epsilon$, then $f(x) \rightarrow 0$ as $x \rightarrow q_{2}$ on the arc $p_{2} q_{2}$.

Let $H$ be a closed 2-cell such that $U \supset H \supset q_{1}$, and $H$ contains a neighborhood of $q_{1}$. By Lemma 4 there exists an $\epsilon_{1}>0$ such that if $c$ is a point and $\rho\left(c, q_{1}\right)<\epsilon_{1}$, then there does not exist an arc of diameter less than $\epsilon_{1}$ lying in $K$ and joining $c$ to $s(c)$. Let $V$ denote an open set containing $q_{1}$ and lying in $H \cdot S\left(q_{1}, \epsilon_{1}\right)$. Since $f$ is not continuous at $q_{1}$, there is a point $c$ in $L \cdot V$ such that $f(c)<\epsilon$. There is an arc $c q_{1}$ in $V$. Then all the hypotheses of Lemma 6 are satisfied. Hence there is an arc $c q_{1}$ lying in $V \cdot L+q_{1}$. Then on this arc $f(x) \rightarrow 0$ as $x \rightarrow q_{1}$ ( since $\left.f(c)<\epsilon\right)$.

Let $\epsilon_{2}$ be the smaller of $\rho\left(p_{1}, q_{1}\right)$ and $\rho\left(c, q_{1}\right)$. Let $H, V, q_{1}, c q_{1}$, and $\epsilon_{2}$, respectively, play the roles of $H, V, p_{1}, c p_{1}$, and $\epsilon$ in Lemma 5 , and let $J$ be the corresponding arc or simple closed curve having properties (2) and (3) of Lemma 5. Then $J$ separates $p_{1}$ and $q_{1}$ and also $c$ and $q_{1}$. Then for $x \varepsilon J$, $x \varepsilon K$ we have $f(x)<\epsilon$. But for $x$ on $p_{1} q_{1}$ we have $f(x)>3 \epsilon$. This is a contradiction, and the theorem is proved.

THEOREM 3. If $q$ is a limit point of $M-K$, then there is a positive number $\epsilon_{1}$ such that there does not exist, for any point $c$, an arc joining $c$ to $s(c)$ lying in $K$ and in $S\left(q, \epsilon_{1}\right)$.

Proof. Let $H$ denote a closed 2-cell in $M$ which contains a neighborhood of $q$. Let $V$ be any open set containing $q$ and let $\epsilon$ be any positive number. Then with the help of Theorem 2 it follows immediately that $q, H, V$, and $\epsilon$ have the properties stated in the hypothesis of Lemma 4 . The number $\epsilon_{1}$ given in the conclusion of Lemma 4 has the required property.

THEOREM 4. If $R$ is a component of $K$ and $q$ is on the boundary of $R$, then $q$ is arc-wise accessible from $R$.

Proof. If $q$ is not a limit point of $M-K$, the result is obvious. If $q$ is a limit point of $M-K$, then it is possible, with the help of Theorems 2 and 3 , to define $H, V, c q$, having properties as stated in the hypothesis of Lemma 6 , with the additional hypothesis that $c \varepsilon R$. Then the arc $c q$ given by Lemma 6 will lie in $R+q$. 
TheORem 5. The set $L$ is identical with $K$.

Proof. It is sufficient to show that if $p$ is in $K$, then $s(p)$ is in $K$. This result was shown in Lemma 1 except for the case where $p$ is on a bounding curve of $M$. Suppose then that $p$ is in $K$ and on a bounding curve of $M$. There is a simple closed curve $J$ bounding an open 2 -cell $H$, such that (1) $J$ is the sum of an arc $a p b$ on that bounding curve of $M$ which contains $p$, and an arc $a q b$ having only $a$ and $b$ on the boundary of $M$, and (2) $\bar{H} \cdot s(\bar{H})=0$. Then the transformation $s$ is topological over $\bar{H}$, whence $s(\bar{H})$ is a closed 2-cell having $s(q)$ on its boundary. Let $c p$ be an arc in $H+p$. Then $s(c p)$ is an $\operatorname{arc} c^{\prime} p^{\prime}$ in $s(H)+p^{\prime}$ and by Lemma $1, s(H)$ is in $L$, hence in $K$. Suppose that $p^{\prime}$ is not in $K$. Then, by Theorem $2, f(x) \rightarrow 0$ as $x \rightarrow p^{\prime}$ on the arc $c^{\prime} p^{\prime}$. But $f(x)=f(s(x))$, and as $x \rightarrow p^{\prime}$ on the $\operatorname{arc} c^{\prime} p^{\prime}$ the point $s(x) \rightarrow p$ on the $\operatorname{arc} c p$, and $f(s(x)) \rightarrow f(p)$ $\neq 0$. This contradiction shows that $p^{\prime}$ is in $K$.

Lемма 7. If an integral subset of $K$ is the sum of two mutually separated connected sets $R_{1}$ and $R_{2}$, then either $s\left(R_{1}\right)=R_{2}$ or else $s\left(R_{1}\right)=R_{1}$.

This lemma follows immediately from the facts that the continuous image of a connected set is connected, and that $s$ is of period 2 ; i.e., that $s(s(A))=A$.

THEOREM 6. The set $K$ has at most two components. If it has two components $R_{1}$ and $R_{2}$, then $s\left(R_{1}\right)=R_{2}$, and $R_{1}$ and $R_{2}$ have the same boundary.

Proof. Let $R_{1}$ be a component of $K$. Then $s\left(R_{1}\right)$ is also a component of $K$. If $q$ is on the boundary of $R_{1}$, then there is an arc $p q$ in $R_{1}+q$. On this arc $f(x) \rightarrow 0$ as $x \rightarrow q$ (Theorem 2). Hence $s(p q-q)+q$ is an arc $p^{\prime} q$ in $s\left(R_{1}\right)+q$, and therefore $q$ is on the boundary of $s\left(R_{1}\right)$. Similarly, every boundary point of $s\left(R_{1}\right)$ is on the boundary of $R_{1}$. The proof will be completed by showing that $\bar{R}_{1}+s\left(R_{1}\right)=M$.

Let $N=\bar{R}_{1}+s\left(R_{1}\right)$, and suppose that there exists a point $t$ in $M-N$. There is an arc $t q$ having only $q$ in the closed point set $N$. Clearly $q$ is on the boundary of $R_{1}$. Furthermore, $q$ is a limit point of $M-K$, for if it were an isolated point in this set then some point of $R_{1}$ could be joined to $t$ by an arc not hitting the boundary of $R_{1}$. Then we can apply Lemmas 4 and 5 and get a closed point set $J$ which separates $t$ from $q$, and such that $J$ is a subset of $R_{1}+s\left(R_{1}\right)$, except possibly for one or two points on the boundary of $R_{1}$. Then $J \cdot t q=0$, since $q$ is the only point of $N$ on $t q$. This contradicts the fact that $J$ separates $t$ and $q$.

LEмma 8. If $p$ is any point of $M-K$, then for every $\epsilon>0$ there is an open set $W \supset p$ such that

(1) $\bar{W}$ is a closed 2-cell of diameter less than $\epsilon$;

(2) $J$, the boundary of $W$ with respect to $M$, is a simple closed curve or an arc with both end-points on a single bounding curve of $M$; and

(3) $J \cdot(M-K)$ consists of 0,1 , or 2 points. 
If $p$ is not a limit point of $M-K$, then the result is obvious. If $p$ is a limit point of $M-K$, then the proof results from an application of Lemmas 4 and 5 . The sets $W$ and $J$ given by Lemma 5 have the desired properties.

Theorem 7 Let $N$ be a component of $M-K$. Then $N$ is a point, an arc, or a simple closed curve, and no point of $N$ is a limit point of $M-K-N$.

Proof. It follows from Lemma 8 that every point of the closed and compact set $M-K$ is of Menger order 0,1 , or 2 with respect to this set. Hence each component of $M-K$ is a point, an arc, or a simple closed curve. Let $q$ be a point of a component $N$ of $M-K$, and suppose that $q$ is a limit point of $M-K-N$. We can apply Lemma 5 and get an open set $W$, with boundary $J$, having properties (1), (2), and (3) of Lemma 5, and the property that no arc $c c^{\prime}$ exists in $K \cdot \bar{W}$, for any point $c$. We suppose that the set $\bar{W} \cdot(M-K)$ $=T_{1}+T_{2}$, mutually separated sets. Then there exists a point $u$ such that $u$ and $u^{\prime}$ are in $K$ and in $\bar{W}^{0}$ (the open 2-cell whose closure is $\bar{W}$ ). Then there exist two arcs $a u b$ and $a u^{\prime} b$ such that (1) $a$ and $b$ are in $T_{1}$ and $T_{2}$, respectively, (2) $s(a u b-a-b)=a u^{\prime} b-a-b$, and (3) $\left(a u b+a u^{\prime} b\right)-a-b$ is in $K \cdot \bar{W}^{0}$. Then $a u b+a u^{\prime} b$ is a simple closed curve $J_{1}$ bounding an open 2-cell $W_{1}$. Neither of the mutually exclusive closed sets $T_{1} \cdot \bar{W}_{1}$ and $T_{2} \cdot \bar{W}_{1}$ separates $u$ from $u^{\prime}$ in $\bar{W}_{1}$, but their sum does. But this is impossible. Hence $(M-K) \cdot \bar{W}$ is connected. Since it contains $q$ it is in $N$. Then $q$ is not a limit point of $M-K-N$.

THEOREM 8. If a component $N$ of $M-K$ is an arc, then each of its end-points, but no other point, is on a boundary curve of $M$; if $N$ is a point or a simple closed curve, then no point of $N$ is on any boundary curve of $M$.

Proof. If $p$ is an end-point of an arc $N$ which is a component of $M-K$, then by Lemma $4 M-K$ locally separates $M$ at $p$, and hence by Theorem 7 $N$ locally separates $M$ at $p$. But this is impossible if $p$ has an open 2-cell neighborhood in $M$. Hence $p$ is on a bounding curve of $M$.

Let $p$ be a point of order 2 on some component $N$ of $M-K$, and let $a p b$ be an arc which is a subset of $N$. There is an arc $a q b$ having only $a$ and $b$ in $M-K$ and such that the simple closed curve $a q b+a p b$ bounds an open 2-cell $U$ which is in $K$. Then it follows that $U+s(U)+a p b$ contains an open 2-cell containing $p$.

In a similar way it can be shown that if the point $p$ is a component of $M-K$, then it lies in an open 2-cell.

\section{PART II}

Lemma 1. If $\lim \sup A_{n}=A$, then $\lim \sup s\left(A_{n}\right) \subset A+s(A)$.

Proof. Let $p$ be a point of $\lim \sup s\left(A_{n}\right)$. Then there exists a sequence of points $\left\{p_{n}\right\}, p_{n} \varepsilon s\left(A_{n}\right)(n=1,2, \cdots)$ such that $p$ is a sequential limit point of $\left\{p_{n}\right\}$. Since $G$ is upper semi-continuous, the sequence $\left\{s\left(p_{n}\right)\right\}$, or some sub- 
quence, has as limit point either $p$ or $s(p)$. But clearly $s\left(p_{n}\right) \varepsilon A_{n}$, since $p_{n} \varepsilon s\left(A_{n}\right)$. Hence if $p$ is a limit point of $\left\{s\left(p_{n}\right)\right\}$, then $p \varepsilon \lim \sup A_{n}$. If $s(p)$ is a limit point of $\left\{s\left(p_{n}\right)\right\}, s(p) \varepsilon \lim \sup A_{n}=A$ and $p \varepsilon s(A)$. In either case the lemma is proved.

Lemma 2. Given $\epsilon>0$, there exists $\delta>0$ such that if $A$ is a point set of $d i$ ameter less than $\delta$, then $s(A)$ is the sum of two sets, each of diameter less than $\epsilon$.

Proof. Suppose the lemma is false. Then there exists an $\epsilon>0$ and a sequence of sets, $\left\{A_{n}\right\}$, such that $d\left(A_{n}\right)<1 / n$, but for no $n$ can $s\left(A_{n}\right)$ be expressed as the sum of two sets, each of diameter less than $\epsilon$. Let $\left\{A_{n_{i}}\right\}$ be a subsequence of $\left\{A_{n}\right\}$ such that $\lim \sup A_{n_{i}}=\lim \inf A_{n_{i}}=p$, a point. Then by Lemma $1, \lim \sup s\left(A_{n_{i}}\right) \subset p+s(p)$. But then clearly, for $n_{i}$ large enough, $s\left(A_{n_{i}}\right) \subset s(p, \epsilon / 2)+s(s(p), \epsilon / 2)$, and thus $s\left(A_{n_{i}}\right)$ is the sum of two sets, each of diameter less than $\epsilon$. This contradiction proves the lemma.

We now have as a corollary of Lemma 2 the following:

Lemma 3. Given $\epsilon>0$, there exists $\delta>0$ such that if $d(A)<\delta$ and $s(A)$ is connected, then $d(s(A))<\epsilon$.

LEMMA 4. If $p$ is a point of $M-K$ and if $q_{1} p, q_{2} p, q_{3} p$ and $q_{4} p$ are arcs in $K+p$ with only the point $p$ in common and such that $s\left(q_{1} p-p\right)=q_{2} p-p$ and $s\left(q_{3} p-p\right)=q_{4} p-p$, then these arcs have the cyclic order $q_{1} p, q_{3} p, q_{2} p, q_{4} p$ about $p$.

Proof. Suppose the lemma is false. Choose $\epsilon>0$ so that the sphere $S(p, 4 \epsilon)$ is an open 2-cell which contains no $q_{i}(i=1,2,3,4)$ and intersects no component of $M-K$ other than the one to which $p$ belongs. Then for every $n$ we can find a point $b_{n}$ of $q_{1} p$ and an arc $b_{n} b_{n}{ }^{\prime}$ in $K$ such that $\rho\left(b_{n}, p\right)<\min (1 / n, \epsilon)$ and $d\left(b_{n} \dot{b}_{n}^{\prime}\right)<1 / n$ and $b_{n} b_{n}^{\prime} \cdot \sum_{i=1}^{4} q_{i} p=b_{n}+b_{n}^{\prime}$.

Now $b_{n}^{\prime} b_{n}^{\prime}+s\left(b_{n} b_{n}^{\prime}\right)$ is a connected integral set, and hence by Lemma 3 , for $n>n_{0}, d^{\prime}\left(b_{n} b_{n}^{\prime}+s\left(b_{n} b_{n}^{\prime}\right)\right)<\epsilon$. Now $b_{n} b_{n}^{\prime}+s\left(b_{n} b_{n}^{\prime}\right)$ contains a simple closed curve $J_{n}$, which is an integral subset of $K\left({ }^{9}\right)$. But since, for $n>n_{0}, J_{n} \subset S(p, 4 \epsilon)$ and $J_{n} \cdot \sum_{i=1}^{4} q_{i} p \subset b_{n}+b_{n}^{\prime}$, it follows that $J_{n}$ contains neither $p$ nor any other point of $M-K$ in its interior. Now let $R$ be the component of $K$ which contains $J_{n}$, and let $I_{n}$ be the interior of $J_{n}$. Since $s(R) \cdot R \neq 0$, it follows from Theorem 6 of Part I that $s(R)=R$. For $n$ sufficiently large, it is easy to see that $R-\left(J_{n}+I_{n}\right)$ is connected. Now we cannot have $s\left(J_{n}+I_{n}\right)=J_{n}+I_{n}$, for that would contradict the principal theorem of Transformations. Hence by Lemma 7 of Part I, $s\left(I_{n}\right)=R-\left(J_{n}+I_{n}\right)$. But there is a positive number $\epsilon_{1}$ such that, for $n>n_{1}, d\left(R-\left(J_{n}+I_{n}\right)\right)>\epsilon_{1}$, and hence, by Lemma 3 , a corresponding positive number $\delta$ exists such that $d\left(I_{n}\right)>\delta$, for every $n>n_{1}$. But $\lim d\left(J_{n}\right)=0$, and since $M$ is compact it follows that $\lim d\left(I_{n}\right)=0$. This contradiction proves the lemma.

We are now in a position to prove

${ }^{(9)}$ See the argument early in $\S 8$ of Transformations proving the existence of a simple closed curve. 
THEOREM 1. If $T$ is a 2-to-1 transformation defined over $M$, then $M$ can be so triangulated that the image under $s$ of every $n$-cell $\alpha$ of the triangulation is an $n$-cell of the triangulation different from $\alpha(n=0,1,2)$. Hence $\chi(M)$ is even, and $\chi(T(M))=\chi(M) / 2$.

Proof. It follows from Theorem 5 of Part I that $M-K$ is an integral set. Also, $M-K$ is a compact metric space, and consists of a finite number of simple closed curves $J_{1}, J_{2}, \cdots, J_{n_{1}}$, of isolated points $p_{1}, p_{2}, \cdots, p_{n_{2}}$, and of $\operatorname{arcs} v_{1}, v_{2}, \cdots, v_{n_{3}}$ whose end-points lie on the bounding curves of $M$; and each bounding curve of $M$ contains either two or no points of $M-K$. (This follows easily from Theorems 7 and 8 of Part I.) Moreover, from Theorem 5 of Part I, if $p$ is an isolated point of $M-K$, then $s(p)$ is also an isolated point of $M-K$. Hence $T$ is a 2-to- 1 transformation defined over $M-K$, and the point set $K_{1}$, over which $s$ is continuous relative to $M-K$, is open and dense in $M-K$. In view of this it can be shown $\left({ }^{10}\right)$ that $K_{1} \supset \sum_{i=1}^{n_{2}} p_{i}+\sum_{i=1}^{n_{3}} v_{i}$, and that $J_{i}$ contains either no point or exactly two points, $u_{1 i}$ and $u_{2 i}$, of $M-K-K_{1}$.

Now let $m$ be a positive number such that the distance between any two components of $M-K$ is greater than $m$, and the distance between any two points of $M-K-K_{1}$ is greater than $m$. We now choose four positive numbers $\epsilon_{1}>\epsilon_{2}>\epsilon_{3}>\epsilon_{4}$ with the following properties:

(1) $4 \epsilon_{1}<m$;

(2) if $B$ and $s(B)$ are connected sets and if $d(B)<\epsilon_{2}$, then $d(s(B))<\epsilon_{1}$ (see Lemma 3);

(3) any simple closed curve of $M$ of diameter less than $\epsilon_{3}$ bounds a 2-cell of $M$ of diameter less than $\epsilon_{2}\left({ }^{11}\right)$; and

(4) if $A$ and $s(A)$ are connected sets, and if $d(A)<\epsilon_{4}$, then $d(s(A))<\epsilon_{3}$ (see Lemma 3).

Now consider an isolated point $p_{j}$ of $M-K$. Let $q_{1}$ be a point of $K$ such that $\rho\left(q_{1}, p_{j}\right)<\epsilon_{4}$ and let $\gamma_{1}$ be an arc of diameter less than $\epsilon_{4}$ from $q_{1}$ to $p_{j}$. Let $\gamma_{2}$ be the arc $p_{j}+s\left(\gamma_{1}-p_{j}\right)$. Let $q_{3}$ be a point of $K$ such that $\rho\left(p_{j}, q_{3}\right)<\epsilon_{4}$, and $q_{3} \& \gamma_{1}+\gamma_{2}$. Let $\gamma_{3}$ be an arc of diameter less than $\epsilon_{4}$ from $q_{3}$ to $p_{j}$ such that $\gamma_{3} \cdot\left(\gamma_{1}+\gamma_{2}\right)=p_{j}$. Let $\gamma_{4}$ be the arc $p_{j}+s\left(\gamma_{3}-p_{j}\right)$. Now by Lemma 4 these arcs have the cyclic order, $\gamma_{1}, \gamma_{3}, \gamma_{2}, \gamma_{4}$, about $p$. We now find two points, $r_{1} \varepsilon \gamma_{1}$ and $r_{3} \varepsilon \gamma_{3}$, and two arcs, $\beta_{1}$ from $r_{1}$ to $r_{3}$, and $\beta_{2}$ from $r_{3}$ to $r_{1}^{\prime}$ with the following properties:

(1) $\left(\sum_{i=1}^{4} \gamma_{i}\right)\left(\beta_{1}+\beta_{2}+\beta_{1}^{\prime}+\beta_{2}^{\prime}\right)=r_{1}+r_{3}+r_{1}^{\prime}+r_{3}^{\prime}$; and

(2) if $r_{1}^{\prime}=r_{2}$ and $r_{3}^{\prime}=r_{4}$, and if $\delta_{i}$ denotes the subarc of $\gamma_{i}$ from $p_{j}$ to $r_{i}$ $(i=1,2,3,4)$, then $d\left(\beta_{1}+\delta_{1}+\delta_{3}\right)<\epsilon_{4}$ and $d\left(\beta_{3}+\delta_{3}+\delta_{2}\right)<\epsilon_{4}$. It follows from the definition of $\epsilon_{3}$ and the fact that $\epsilon_{4}<\epsilon_{3}$, that $\beta_{1}+\delta_{1}+\delta_{3}$ and $\beta_{3}+\delta_{3}+\delta_{2}$ are simple closed curves which are the boundaries of closed 2-cells, $\lambda_{1}$ and $\lambda_{3}$,

(10) $K_{1}$ will be used to denote this set throughout the rest of the paper. The proofs of the statements of this sentence, while not trivial, are sufficiently straightforward to be omitted.

(11) It is easy to see that, since $M$ is compact, $\epsilon_{3}$ can be chosen to satisfy this property. 
respectively. It is easy to see that $s\left(\beta_{1}\right)$ is an arc $\beta_{2}$ from $r_{2}$ to $r_{4}$, and $s\left(\beta_{3}\right)$ is an arc $\beta_{4}$ from $r_{4}$ to $r_{1}$; and further, that $s\left(\lambda_{1}^{0}\right)$ is an open 2 -cell $\lambda_{2}^{0}$ bounded by $\beta_{2}+\delta_{2}+\delta_{4}$, and $s\left(\lambda_{3}^{0}\right)$ is an open 2-cell $\lambda_{4}^{0}$ bounded by $\beta_{4}+\delta_{4}+\delta_{1}$. Thus the neighborhood of $p_{j}$ has been triangulated in accordance with the theorem.

We suppose that this has been done for every $p_{j}\left(j=1,2, \cdots, n_{1}\right)$. In a similar manner we triangulate the neighborhood of each of the simple closed curves $J_{1}, J_{2}, \cdots, J_{n_{1}}$ and each of the arcs $v_{1}, v_{2}, \cdots, v_{n_{3}}$ in such a way that these simple closed curves and arcs appear in the triangulation as sums of 1 -cells and vertices of the triangulation which map under $s$ into 1-cells and vertices of the triangulation. We take care, also, to make every point of $M-K-K_{1}$ a vertex of the triangulation.

We now let $A_{1}$ denote the complex which is composed of all these neighborhoods of the components of $M-K$ so triangulated, and let $H=M-A_{1}^{*}$. Then $\bar{H}$ is a closed and compact point set over which $f$ is continuous and positive. Hence there is a positive number $\psi$ such that $f(x)>2 \psi$ if $x \varepsilon \bar{H}$. Now we choose three positive numbers $\psi_{1}, \psi_{2}$, and $\psi_{3}$ as follows: (1) if $\gamma$ is a simple closed curve of diameter less than $4 \psi_{1}$, then $\gamma$ bounds a 2 -cell of diameter less than $\psi$ (this implies $4 \psi_{1}<\psi$ ); and (2) if $B$ and $s(B)$ are connected sets, then if $d(B)<\psi_{2}$, it follows that $d(s(B))<\psi_{1}$; and if $d(B)<\psi_{3}$, it follows that $d(s(B))<\psi_{2}$. (See Lemma 3. These conditions imply $\psi_{1}>\psi_{2}>\psi_{3}$.) If $A_{1}$ contains a 1-cell $\alpha$ such that $d(\alpha) \geqq \psi_{3}$, let $A_{2}$ be a subdivision of $A_{1}$ containing no such 1-cell but still having the property that the image under $s$ of an $n$-cell of $A_{2}$ is an $n$-cell of $A_{2}(n=0,1,2)$. If $A_{1}$ contains no such 1 -cell, $A_{2}=A_{1}$.

Now it may be possible to find either one, two, or three arcs whose endpoints are vertices of $A_{2}$ but which otherwise lie in $M-A_{2}{ }^{*}$ and which, together with two, one, or no arcs, respectively, of $A_{2}{ }^{*}$, bound a 2 -cell $\phi$ of diameter less than $\psi$ whose interior lies in $M-A_{2}{ }^{*}$, but not in $S\left(A_{2}{ }^{*}, \psi_{3}\right)$. If such a possibility exists, we add to $A_{2}$ this 2-cell $\phi$ and also $s(\phi)$. Since $d(\phi)<\psi, \phi \cdot s(\phi)=0$. After extending $A_{2}$ in this manner as many times as possible, successively, we call the extended complex $A_{3}$.

If $M-A_{3}^{*}$ contains an open 2-cell $\alpha^{0}$ such that the boundary of $\alpha^{0}$ consists of three vertices and three 1-cells of $A_{3}$ and $s\left(\alpha^{0}\right) \cdot \alpha^{0}=0$, then we add $\alpha^{0}$ and $s\left(\alpha^{0}\right)$ to $A_{3}$. After adding all such 2-cells to $A_{3}$, we call the new complex $A_{4}$. We then obtain $A_{5}$ from $A_{4}$ by subdivision, in the same way that we obtained $A_{2}$ from $A_{1}$.

Now let $r_{1}, r_{2}, \cdots, r_{k}$ denote the vertices of $A_{5}$ which are on the boundary of $M-A_{5}{ }^{*}$. Let $r_{k+1}$ be a point of $M-A_{5}^{*}$ for which $\psi_{3}<\rho\left(r_{k+1}, A_{5}^{*}\right)<\psi_{2}$. Join $r_{k+1}$ to two points, $r_{i_{2}}$ and $r_{i_{3}}$, which are end-points of the same 1-cell $\alpha_{1}$ of $A_{5}$ by arcs $\alpha_{2}$ and $\alpha_{3}$ in such a way that (1) $\alpha_{2} \cdot \alpha_{3}=r_{k+1}$, (2) $\alpha_{2} \cdot A_{5}{ }^{*}=r_{i_{2}}$ and $\alpha_{3} \cdot A_{5}{ }^{*}=r_{i_{3}}$, and (3) $d\left(\alpha_{i}\right)<2 \psi_{1}(i=1,2)$. To see that this is possible, we draw an $\operatorname{arc} \beta$ of diameter less than $\psi_{2}\left({ }^{12}\right)$, from $r_{k+1}$ to some point $p$ in the interior

(12) This is possible since the metric $\rho$ which we are using has the property that if $H$ is closed and $\rho(x, H)<\epsilon$, then there exists an arc from $x$ to a point of $H$ of diameter less than $\epsilon$. 
of a 1 -cell $\alpha_{1}$ on the boundary of $A_{5}{ }^{*}$. Since no 1 -cell of $A_{5}$ has diameter as great as $\psi_{3}$, we can draw an arc in $M-A_{5}{ }^{*}$ from $r_{k+1}$, running along very close to $\beta$ and then running along close to $\alpha_{1}$ until we get near an end-point $r_{i_{2}}$ of $\alpha_{1}$. Then we run our arc into $r_{i,}$ and call the arc $\alpha_{2}$. Similarly, on the other side of $\beta$, we draw $\alpha_{3}$ from $r_{k+1}$ to $r_{i_{3}}$. If in drawing these arcs we stay close enough to $\beta$ and then to $\alpha_{1}$, neither $\alpha_{2}$ nor $\alpha_{3}$ can have diameter greater than $\psi_{2}+\psi_{3}$, which is less than $2 \psi_{1}$. Moreover, $\alpha_{1}+\alpha_{2}+\alpha_{3}$ is a simple closed curve of diameter less than $4 \psi_{1}$, and hence by definition of $\psi_{1}$ bounds a 2 -cell $\eta$ of diameter less than $\psi$. It follows that $s(\eta)$ is a 2-cell such that $\eta \cdot s(\eta)=0$ and $s(\eta) \cdot A_{5}=s\left(\alpha_{1}\right)$. We add $\eta$ and $s(\eta)$ to $A_{5}$ and call the resulting complex $A_{6}$.

We now begin over again, obtaining $A_{7}$ from $A_{6}$ in the same way that we obtained $A_{2}$ from $A_{1}$, etc. It is clear that the method of extension from $A_{5}$ to $A_{6}$ can be carried out only a finite number of times. For otherwise $M$ would contain an infinite point set with no limit point, since we require that the new vertex have a distance greater than $\psi_{3}$ from the point set covered by the complex. But when this method of extension cannot be repeated that means that the complex we have, $A_{n}$, has the property that there is no point of $M-A_{n}{ }^{*}$ which has a distance greater than $\psi_{3}$ from $A_{n}{ }^{*}$. When this is the case, it is easy to see that a finite number of applications of the methods of extension from $A_{2}$ to $A_{3}$ and from $A_{3}$ to $A_{4}$ will give a complex which covers $M$. Hence the theorem is proved.

LEMMA 5. Let $p$ be an interior point of $M$. If $p$ is neither an isolated point of $M-K$, nor a point of $M-K-K_{1}$, then $T(p)$ has a 2-cell neighborhood in $T(M)$. Otherwise $T(p)$ has a neighborhood in $T(M)$ which is homeomorphic to a neighborhood of the vertex of the double cone $x^{2}=y^{2}+z^{2}$.

Hereafter when we speak of a manifold with identifications we shall always mean by "identifications" a finite number of points with neighborhoods homeomorphic to a neighborhood of the vertex of the double cone.

Proof. If $p$ belongs to $K$, the result follows immediately; for $s$ is locally a homeomorphism at every point of $K$, and if $U$ is an open subset of $K$ such that $U \cdot s(U)=0$, then $s(U)$ and $T(U)$ are homeomorphic. But this means that $T(U)$ contains an open set which is an open 2-cell containing $T(p)$.

If $p$ belongs to $(M-K) \cdot K_{1}$, but is not an isolated point of $M-K$, then $p$ belongs to an arc or a simple closed curve of $M-K$. Let $c$ denote the arc or the simple closed curve. By triangulating the neighborhood of $p$ and of $s(p)$ in the same way in which they were triangulated in the proof of Theorem 1 , and taking care to have neither $p$ nor $s(p)$ be a vertex in this triangulation, we obtain mutually exclusive open 2-cells $V$ and $W$ containing $p$ and $s(p)$, respectively, and having the following properties:

(1) $V-c=V_{1}+V_{2}$, mutually separated open 2-cells such that $s\left(V_{1}\right)=V_{2}$;

(2) $V \cdot c$ is an open 1 -cell lying in $K_{1}$;

(3) $V \cdot(M-K)=V \cdot c$; 
(4) $W \cdot s(V \cdot c)=W \cdot(M-K)$;

(5) $W-s(V \cdot c)=W_{1}+W_{2}$, mutually separated open 2-cells such that $s\left(W_{1}\right)=W_{2}$.

It follows that $s(V \cdot c)$ is an open 1-cell, and that $T(V \cdot c)=T(s(V \cdot c))$ is an open 1-cell. Likewise, each of the sets $T(V)$ and $T(W)$ is homeomorphic to the intersection of the interior of the unit sphere in the Euclidean plane with the half-plane $x \geqq 0$. Moreover, $T(V) \cdot T(W)=T(V \cdot c)$, an open 1-cell. Hence $T(V+W)$ is an open 2-cell containing $T(p)$. Furthermore, it is clear that $T(V+W)$ is an open set in $T(M)$, since $V+W$ is an integral open set in $M$.

If $p$ is an isolated point of $M-K$, then, as we remarked in the proof of Theorem $1, s(p)$ is also. We triangulate the neighborhood of $p$ and of $s(p)$ as we did in the proof of Theorem 1. From the description of that triangulation, it is easy to verify that if $\pi(p)$ denotes the open 2-cell containing $p$ whose closure is $\lambda_{1}+\lambda_{2}+\lambda_{3}+\lambda_{4}$ (see the proof of Theorem 1 for the meaning of $\left.\lambda_{i}\right)$, then $T(\pi(p))$ is an open 2-cell. Similarly for $\pi\left(p^{\prime}\right)$. But $T(\pi(p)) \cdot T\left(\pi\left(p^{\prime}\right)\right)=T(p)$. Hence $T\left(\pi(p)+\pi\left(p^{\prime}\right)\right)$ is homeomorphic to a neighborhood of the vertex of the double cone $x^{2}=y^{2}+z^{2}$, and since $\pi(p)+\pi\left(p^{\prime}\right)$ is an integral open set in $M, T\left(\pi(p)+\pi\left(p^{\prime}\right)\right)$ is open in $T(M)$, and the lemma follows for this case.

The case in which $p$ belongs to $M-K-K_{1}$ is handled in a somewhat similar manner.

LEMMA 6. Let $p$ be on one of the boundary curves of $M$. Then $T(p)$ has a neighborhood in $T(M)$ which is homeomorphic to a neighborhood of $p$ in $M$.

The proof follows in much the same way that the proof of the preceding lemma followed.

THEOREM 2. If $M$ is a compact manifold, then $T(M)$ is a compact manifold or can be obtained from a compact manifold by the identification by pairs of a finite number of points. If $M$ is a compact manifold with boundary, then $T(M)$ is a compact manifold with.boundary or can be obtained from a compact manifold with boundary by the identification by pairs of a finite number of interior points.

Proof. The first statement follows as a corollary of Lemma 5. To prove the second statement, we first show that if $c$ is a boundary curve of $M$, then $T(c)$ is a simple closed curve. For suppose first that $K \triangleright c$. Then by Theorem 8 of Part I, $c \cdot K \neq 0$. Let $p$ be a point of $c \cdot K$, and let $p q$ be a subarc of $c$ which lies in $K+q$ but not in $K$. Then $p^{\prime} q=s(p q-p)+q$ is a subarc of $c$, by virtue of Theorem 2 of Part I and the fact that the image under $s$ of a boundary point of $M$ is a boundary point of $M$. Let $p r$ be a subarc of $c$ such that $p r \cdot p q=p$ and $K+r \supset p r$ but $K \triangleright p r$. (It is easy to see that there must be a point $r \neq q$ such that $r \varepsilon c \cdot(M-K)$. For otherwise, as a variable point $x$ 
moved continuously along $c-p q-p^{\prime} q+p$ from $p, s(x)$ would move continuously along $c-p q-p^{\prime} q+p^{\prime}$ and a two-to-one transformation would be defined on the arc $c-p q-p^{\prime} q+p+p^{\prime}$, in contradiction to the result of $\mathrm{O}$. G. Harrold ( $\left.{ }^{1}\right)$.) Then as above, $p^{\prime} r=s(p r-r)+r$ is a subarc of $c$. Then clearly $c=p q+p^{\prime} q+p r+p^{\prime} r$, and a direct argument shows that $s(q)=r$. Hence in this case $T(c)$ is a simple closed curve.

Now suppose that $K \supset c$. Then $s(c)$ is a simple closed curve which is a boundary curve of $M$, and hence either $s(c)=c$ or else $s(c) \cdot c=0$. In either case $T(c)$ is a simple closed curve.

Now by combining the fact that $T(c)$ is a simple closed curve with Lemma 5 and Lemma 6, we see that the second statement of our theorem is proved.

Moreover, it is easy to see that we have also proved

Lemma 7. If $M$ has $n$ boundary curves, then $T(M)$ has $k$ boundary curves, where $n / 2 \leqq k \leqq n$.

We now prove

THEOREM 3. Given a space $M$ and a space $B_{k}$, a necessary and sufficient condition that there exist a 2-to-1 transformation $T$ such that $T(M)=B_{k}$ is that $M$ and $B_{k}$ be properly related. (See the introduction.)

Proof. The necessity follows immediately from Theorems 1 and 2 and Lemmas 5,6 , and 7 . The sufficiency will be proved by actually constructing the transformation $T$.

Let $M$ be an orientable manifold, and let $M$ be embedded in Euclidean 3 -space in such a way that $M$ is symmetric with respect to the $x y$-plane, and the common part of $M$ and the $x y$-plane consists of $h$ simple closed curves $c_{1}, c_{2}, \cdots, c_{h}$ if $\chi(M)=2(h-2)$. If $p$ is a point of $M$ not in the $x y$-plane, we define $s(p)$ as the reflection of $p$ in the $x y$-plane. If $B_{k}$ is an orientable manifold, then $h$ is even and we let $s$ map $c_{1}$ into $c_{2}, c_{3}$ into $c_{4}, \cdots, c_{h-1}$ into $c_{h}$ topologically. When $s$ is defined, $T$ is determined, and the theorem is proved for this case. If $B_{k}$ is a non-orientable manifold, we define $s$ exactly as before except that $s$ maps $c_{h}$ into itself by identifying diametrically opposite points, in case $h$ is odd, and it maps both $c_{h}$ and $c_{h-1}$ in to themselves in this manner if $h$ is even. If $B_{k}$ is an orientable manifold with $b$ identifications, then $h-b$ is even and non-negative. For if a manifold $N$ has $\chi(N)=v$, and if $N_{1}$ is obtained from $N$ by identifying $b$ pairs of points, then $\chi\left(N_{1}\right)=v+b$. Hence we define $s(p)$ as before for a point $p$ not in the $x y$-plane, and we let $x$ map $c_{i}$ $(i=1,2, \cdots, b)$ into itself by a reflection in a diameter and the identification of the two points of $c_{i}$ which lie on the diameter. And we let $s$ map $c_{b+1}$ into $c_{b+2}, c_{b+3}$ into $c_{b+4}, \cdots, c_{h-1}$ into $c_{h}$, topologically. If $B_{k}$ is a non-orientable manifold with $b$ identifications, $s$ is defined as in the preceding case except that if $h-b$ is odd, then $s$ maps $c_{h}$ into itself by identifying diametrically 
opposite points, and if $h-b$ is even then $s$ maps both $c_{h-1}$ and $c_{h}$ into themselves in that manner. This completes the cases in which $M$ is an orientable manifold.

If $M$ is a non-orientable manifold, and $\chi(M)$ is even, then either $M$ is a Klein's bottle, in which case $\chi(M)=0$, or else $M$ can be obtained from a Klein's bottle by inserting $h$ handles, in which case $\chi(M)=2 h$. If $M$ is a Klein's bottle, let $M$ be triangulated as in the figure. The numbers in this figure refer to the vertices by which they are placed and the letters denote the

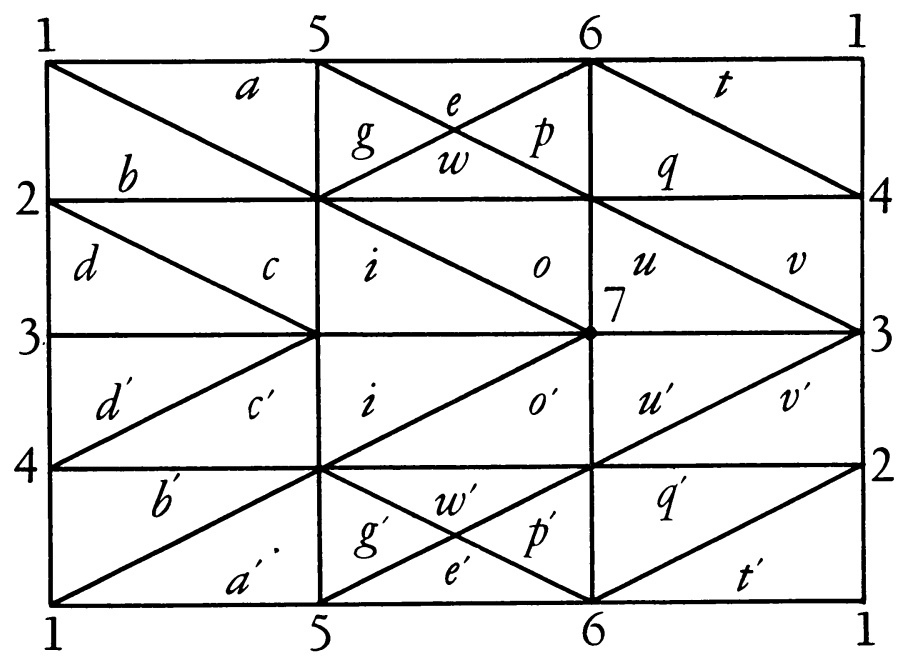

2-cells in which they are placed. The top edge of the figure is identificed with the bottom edge, and the points of the left edge reading up are identified with those of the right edge reading down, as the numbers indicate. Let $s$ map the 2 -cells above the horizontal bisector 373 into those below 373 by reflection in 373 , as the primes indicate. This defines $s$ for every point except those points of the horizontal bisector 373 and of the edge 1561 ; we call these two simple closed curves $c_{1}$ and $c_{2}$. If $B_{k}$ is a torus, we let $s$ map $c_{1}$ in to $c_{2}$ topologically; if $B_{k}$ is a projective plane with one identification we let $s$ map $c_{1}$ into itself by identifying diametrically opposite points and $c_{2}$ into itself by identifying points by reflection in a diameter and identifying the points of $c_{2}$ on the diameter; if $B_{k}$ is a Klein's bottle, we let $s$ map $c_{i}$ into itself by identification of diametrically opposite points $(i=1,2)$; if $B_{k}$ is a sphere with two identifications, we let $s$ map $c_{i}$ into itself by reflection in a diameter and identification of the points of $c_{i}$ on the diameter $(i=1,2)$.

If $M$ is non-orientable and $\chi(M)=2 h>0$, then $M$ can be constructed as follows: Let $N_{1}$ be the manifold with boundary obtained from the figure by deleting the open 2-cells $w$ and $w^{\prime}$, and let $s$ map $N_{1}-\left(c_{1}+c_{2}\right)$ into itself in the manner described above. Let $N_{2}$ be an orientable manifold for which 
$\chi\left(N_{2}\right)=2 h-4$. Let $N_{2}$ be embedded in Euclidean 3-space so that it is symmetric with respect to the $x y$-plane and the common part of $N_{2}$ and the $x y$-plane consists of $h$ simple closed curves. Let $s$ map the points of $N_{2}$ above the $x y$-plane into those below the $x y$-plane by reflection in that plane. Let $\alpha$ be an open 2-cell in $N_{2}$ lying above the $x y$-plane and having a simple closed curve as boundary, and let $\alpha^{\prime}=s(\alpha)$. Delete $\alpha$ and $\alpha^{\prime}$, and identify the boundaries of $\alpha$ and $\alpha^{\prime}$ with the boundaries of $w$ and $w^{\prime}$, respectively. Then we have defined $s$ over $M$ except for $h+2$ simple closed curves. We define $s$ over these simple closed curves, using one of, or a combination of, the three methods already described for defining $s$ over simple closed curves, depending on the character of the image space $B_{k}$.

The definition of $T$ in the cases in which $M$ is a manifold with bounding curves is analogous to its definition in the cases already treated.

Conclusion. It is known that in some cases a space $M$ may be mapped into a space $B$ in a continuous, exactly 2 -to- 1 fashion, in at least two essentially different ways. For example, a sphere may be mapped into a projective plane (1) by identifying diametrically opposite points, or (2) by identifying point pairs which are symmetrical with respect to the equatorial plane, and then identifying diametrically opposite points on the equator. It is easy to show that the two mappings so defined are not topologically equivalent $\left({ }^{13}\right)$. The following problem naturally arises: For $a$ given $M$ and $B$ how many topologically different 2-to-1 continuous mappings of $M$ into $B$ are there? It seems very likely that this number is finite.

(13) For a definition of this term see G. T. Whyburn, Interior transformations on compact sets, Duke Mathematical Journal, vol. 3 (1937), p. 373, footnote 8.

Duke University, Durham, N. C. 